\title{
LES SEDIMENTS D'UN LAC DE HAUTE MONTAGNE : STRUCTURE, NATURE ET PEUPLEMENT
}

\author{
par N. Grani ${ }^{1}$ et C. Lucas ${ }^{2}$.
}

\begin{abstract}
La variation verticale des composantes physiques, chimiques et faunistiques est analysée sur des carottes de sédiment prélevées dans la zone profonde du lac de Port-Bielh (Pyrénées centrales). Ces sédiments sont particulièrement fins $(100 \%<50 \mu)$ et meubles. Leur structure est remaniée par remise en suspension et décantation ainsi que par l'activité des organismes. La nature du sédiment ne reflète pas la composition chimique du bassin versant. Elle traduit une importante migration des ions en solution.

La densité maximale de peuplement se situe dans les couches superficielles mais la répartition verticale varie selon les groupes. Les modalités de cette répartition sont évoquées.
\end{abstract}

\section{The sediments of a high mountain lake : structure, nature and populations.}

The vertical variations of physical and faunistic components in cores of sediment samples from the profundal zone of lake Port-Bielh (Central Pyrences) were analysed. These sediments are particularly fine $(100 \%<50 \mu)$ and muddy. Their structure is formed by constant suspension and decantation as well as through the activity of benthic organisms. The nature of the sediment does not reflect the chemical composition of the surrounding basin, but is the product of an important movement of ions in solution.

The maximal population density is found in the superficial layers but the vertical distribution varies among groups. The modalities of these distributions are deseribed.

Situé dans le massif granitique du Néouvielle (zone axiale des Pyrénées centrales), le lac de Port-Bielh a fait l'objet de nombreuses études concernant le phytoplancton (Capblancq 1973), le benthos (Laville 1971a et b, Giani et Laville 1973, Rey et Dupin 1973a et b, Juget et Giani 1974). Les propriétés physico-chimiques de l'eau sont bien connues depuis les travaux de Capblancq et Laville (1968), mais la sédimentologie du lac n'avait pas encore été abor-

1. Laboratoire d'Hydrobiologie, Université Paul Sabatier, 118, route de Narbonne, 31077 Toulouse-Cédex.

2. Laboratoire de Géologie-Pétrologie, Université Paul Sabatier, 38, rue des 36-Ponts, 31 Toulouse. 
dée. Laville (1971) a cependant souligné l'importance de la nature du substrat sur la répartition des Chironomides dans ce lac.

Dans ce travail nous avons analysé sommairement les caractères structuraux, texturaux et chimiques des sédiments; nous avons également étudié la répartition verticale de la faune dans le substrat.

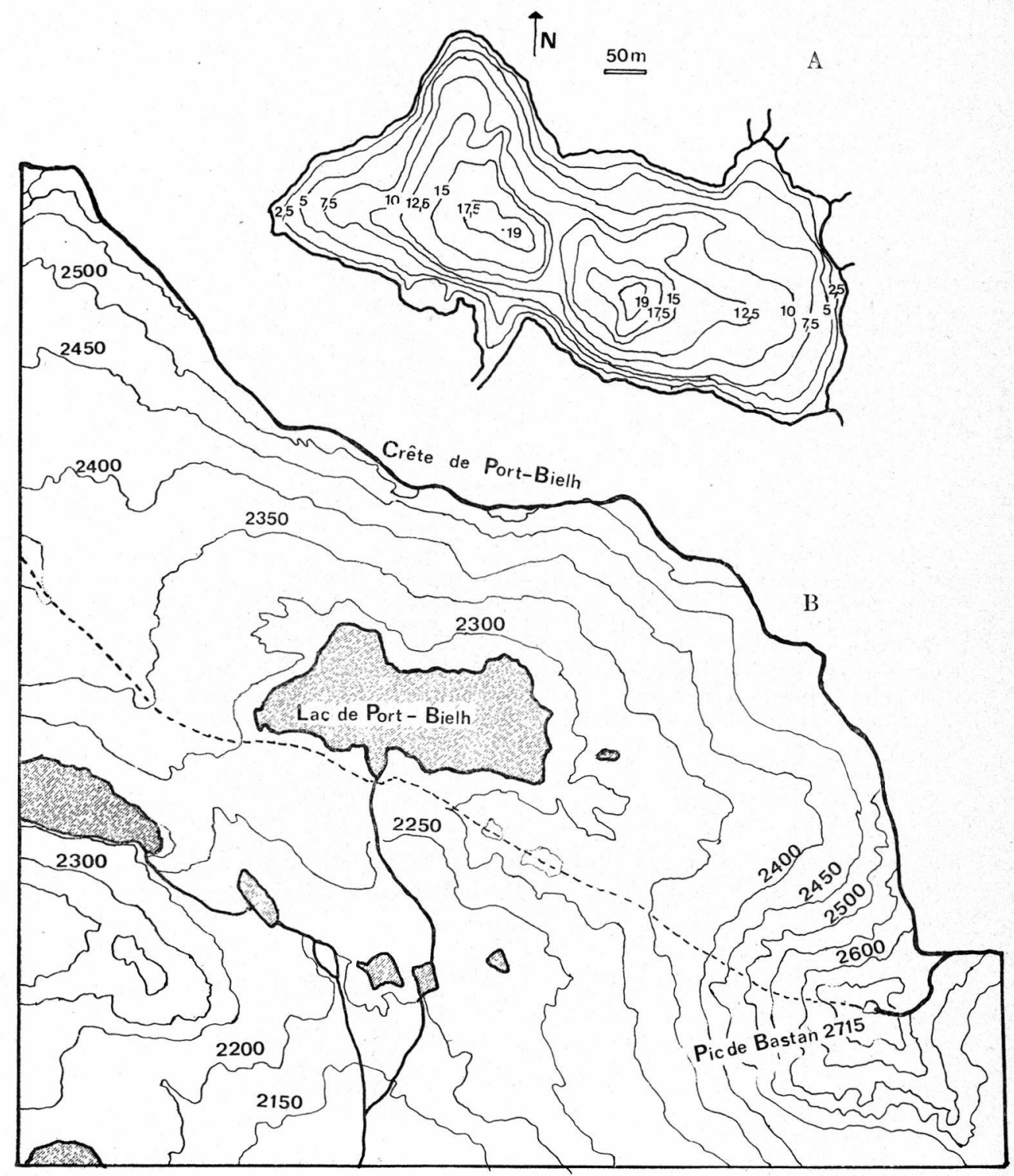

FIG. 1. - 1a : carte du bassin versant du lac de Port-Bielh (échelle : 1/12000). -..- limites du bassin versant; 1 b : carte morphométrique du lac de PortBielh (d'après Capblancq et Laville 1968). 


\section{1. - MILIEU}

A $2280 \mathrm{~m}$ d'altitude, dans un vallon largement ouvert au sud, le lac de Port-Biclh constitue un plan d'eau de 16,5 ha, retenu par un verrou glaciaire. Son bassin versant (fig. 1) est peu étendu puisqu'il ne couvre que 200 ha environ, soit 12 fois la superficie du lac. Il est formé de cônes d'éboulis, de chaos morainiques et d'altération séparés par des pelouses rases à Festukia eskia ou des touffes de Rhododendron ferrugineum. Quelques rares pins à crochets (Pinus uncinata) occupent les mamelons rocheux. Il est essentiellement alimenté par l'eau de fonte des neiges et, en été, par quelques sources d'éboulis à faible débit qui apparaissent près du bord oriental.

Dans le lac, d'une profondeur maximum de $19 \mathrm{~m}$, Capblancq et Laville (1968) ont distingué deux zones : une zone littorale encombrée de gros blocs granitiques ct une zone littori-profonde uniformément recouverte de vase et sur laquelle se développe une prairie dense de Nitella flexilis. Les caux sont faiblement minéralisées mais la conductivité augmente netlement au niveau du fond pendant l'hiver. Ceci semble dû essentiellement à une augmentation des taux de $\mathrm{Ca}^{++}$, de $\mathrm{Mg}^{++}$et du fer en profondeur. Les pH sont toujours basiques. En période de stagnation hivernale, sous la glace, la zone profonde connaît un important déficit en $\mathrm{O}^{2}$.

Les apports détritiques dans le lac de Port-Bielh sont peu abondants vu la nature de son alimentation, la faible élendue et l'aridité de son bassin versant. Toutefois, étant largement ouvert au sud, le bassin de Port-Bielh reçoit, épisodiquement, des apports éoliens africains non négligeables. Ainsi d'après les travaux de Bücher et Lucas (1972) on peut évaluer à plus de $2000 \mathrm{~kg}$ un tel apport en février 1972. Les apports biogéniques autochtones sont réduits vu le caractère oligotrophe de la masse d'eau.

\section{2. - SÊDIMENTOLOGIE DU LAC}

\section{1. - Prélèvements.}

Deux séries d'échantillonnages ont été effectuées, après Ia période d'isothermie d'automne, le 11 et le 26 novembre 1973, à l'aide d'un carottier spécial (Giani 1974). Ces échantillons ont été prélevés à 19 m dans la fosse orientale du lac (fig. 1). Au total, 11 carottes de 50 à $55 \mathrm{~cm}$ de hauteur et de $50,2 \mathrm{~cm}^{2}$ de section ont ainsi été recueillies ; 3 d'entre elles, conservées intactes, nous ont permis d'analyser le sédiment. Les 8 autres ont été immédiatement découpées en tranche de 5 ou $10 \mathrm{~cm}$ le 11-11-1973 et de $5 \mathrm{~cm}$ le 26-11-1973 en vue de l'étude faunistique. 


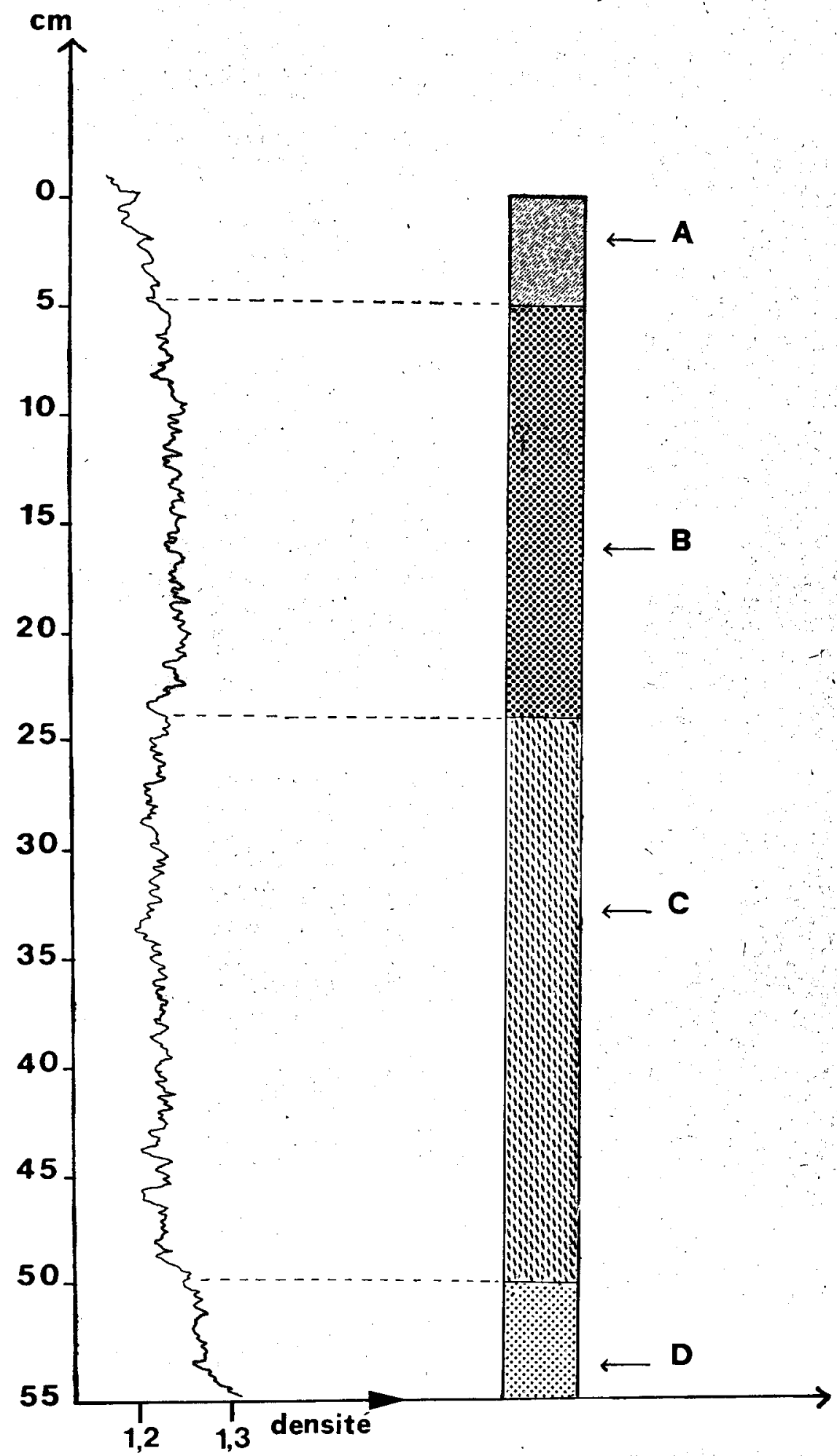

Fig. 2. - Aspect général d'une carotte et densité du sédiment (A, B, C, D niveaux des analyses granulométriques). 


\section{2. - Structure et composition des sédiments.}

L'examen des carottes dès leur émersion révèle quatre zones assez bien différenciées (fig. 2) ; on distingue de la surface vers le bas :

-. une couche de 3 à $5 \mathrm{~cm}$ de couleur gris-rouille;

- une zone de $20 \mathrm{~cm}$, gris-brun, où le sédiment peu tassé a un aspect floconneux ;

- une couche de $25 \mathrm{~cm}$, gris clair, un peu plus compacte ;

- une zone de $5 \mathrm{~cm}$ au moins, gris-vert, où la compaction est plus grande.

La mesure de la densité par gamma-densimétrie (atténuation du rayonnement proportionnelle à la densité) justifie la distinction de ces quatre zones (fig. 2). Il faut noter que la densité est assez homogène dans les $45-50$ premiers centimètres particulièrement meubles; elle augmente au-delà, ce qui correspond à la plus grande compacité du sédiment déjà décelée à l'œil. Il faut souligner les faibles valeurs $(1,2-1,3)$ de cette densité qui traduisent, d'une façon générale, la faible compaction et la teneur en eau très élevée
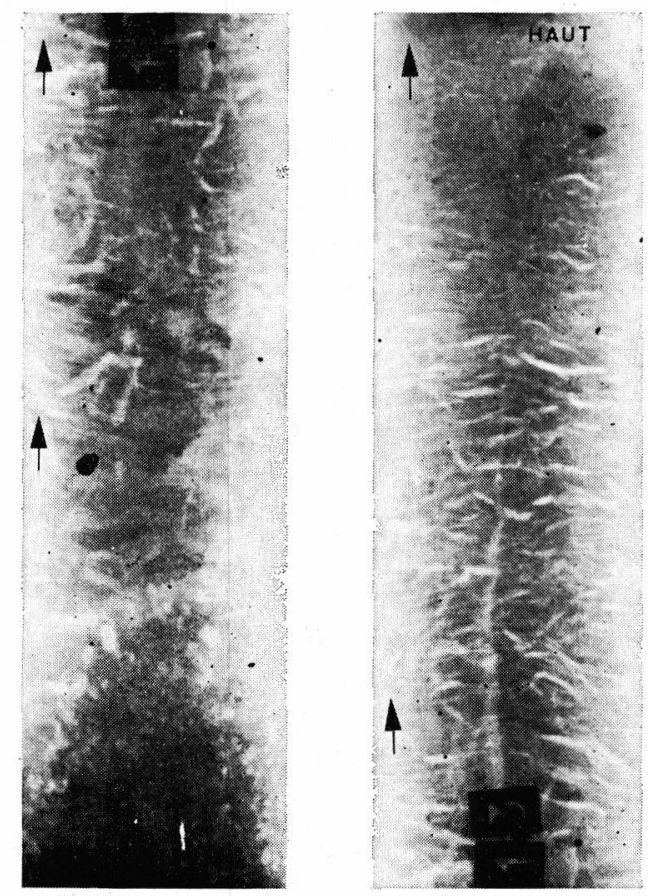

FIG. 3. - Radiographie d'une carotte. 
du sédiment. Le sédiment total, sec, a une densité - mesurée au picnomètre - de l'ordre de 2 à 2,1 .

\section{3. - Granulométrie.}

Les analyses granulométriques ont été effectuées à la pipette d'Andreasen-Rivière après attaque ménagée de la matière organique par $\mathrm{H}^{2} \mathrm{O}^{2}\left(\mathrm{pH}=8,5 ; \mathrm{T}^{\circ}=50{ }^{\circ} \mathrm{C}\right)$ pendant quatre jours. Les résultats sont exprimés sous forme de courbe cumulative (fig. 4). Afin de mieux illustrer l'exceptionnelle finesse de grain des sédiments du lac de Port-Bielh, nous donnons comme terme de comparaison la courbe granulométrique des poussières éoliennes tombées en février 1972 (F, fig. 4). La totalité de la fraction minérale des sédiments considérés est inférieure à 50 microns et la médiane varie entre 0,6 et 1,6 microns alors qu'elle est de 10 microns pour les poussières éoliennes évoquées ci-dessus. Les courbes granulométriques des échantillons $\mathrm{A}$ à $\mathrm{C}$ (fig. 4) reflètent un granoclassement des sédiments dans la tranche supérieure $(\mathrm{de}-50 \mathrm{~cm}$ à la surface) ; la dimension des grains décroît de bas en haut comme dans un dépôt résultant d'une décantation simple.

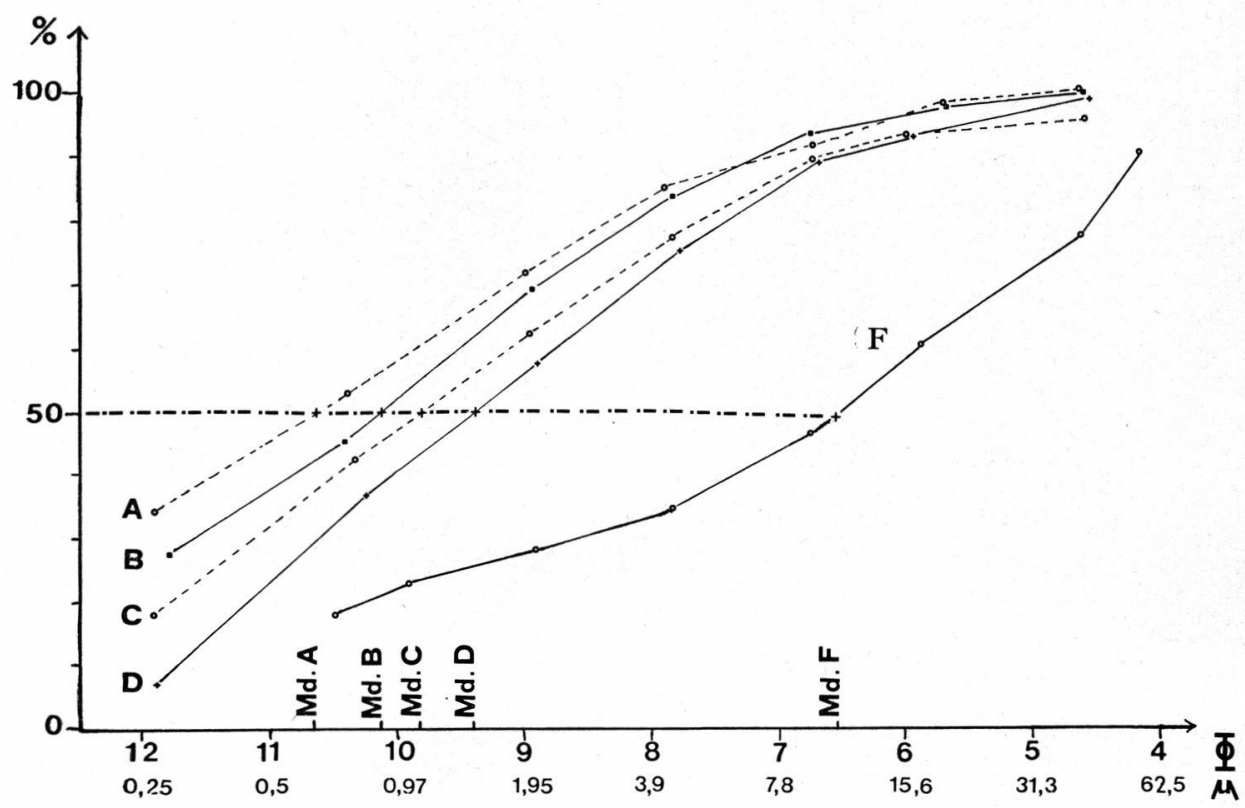

Fig. 4. - Analyses granulométriques des sédiments à quatre niveaux d'une carotte (A, B, C, D voir fig. 2); F : courbe granulométrique des poussières éoliennes tombées en février 1972. 
D'autre part, la radiographie aux rayons $\mathrm{X}$ ne fait pas apparaître de structure laminée particulière (fig. 3) ; elle met en évidence la présence de vacuoles qui pourraient être des bulles de gaz ou des sections de tube d'invertébrés fouisseurs.

Une telle structure, différente de la stratification fine à laquelle on aurait pu s'attendre dans un lac pauvre en apports détritiques implique une remise en suspension du sédiment. Ce phénomène peut être physique, déterminé par le mouvement des eaux lors de la débâcle et des périodes d'isothermie, mais aussi biologique, provoqué par l'activité fouisseuse des organismes benthiques. Il es! encore difficile de préciser ce point; notons simplement que nous n'avons jamais observé de turbidité de l'eau dans les prélèvements effectués en toutes saisons, à toutes les profondeurs. La seule certitude que nous ayons concerne l'activité des organismes, nous y reviendrons ultérieurement.

\section{4. - Minéralogie des sédiments.}

Les terrains granitiques - granodioritiques plus exactement qui constituent le bassin versant sont, dans une grande proportion à l'origine des sédiments du lac (il est encore difficile de faire la part des apports éoliens).

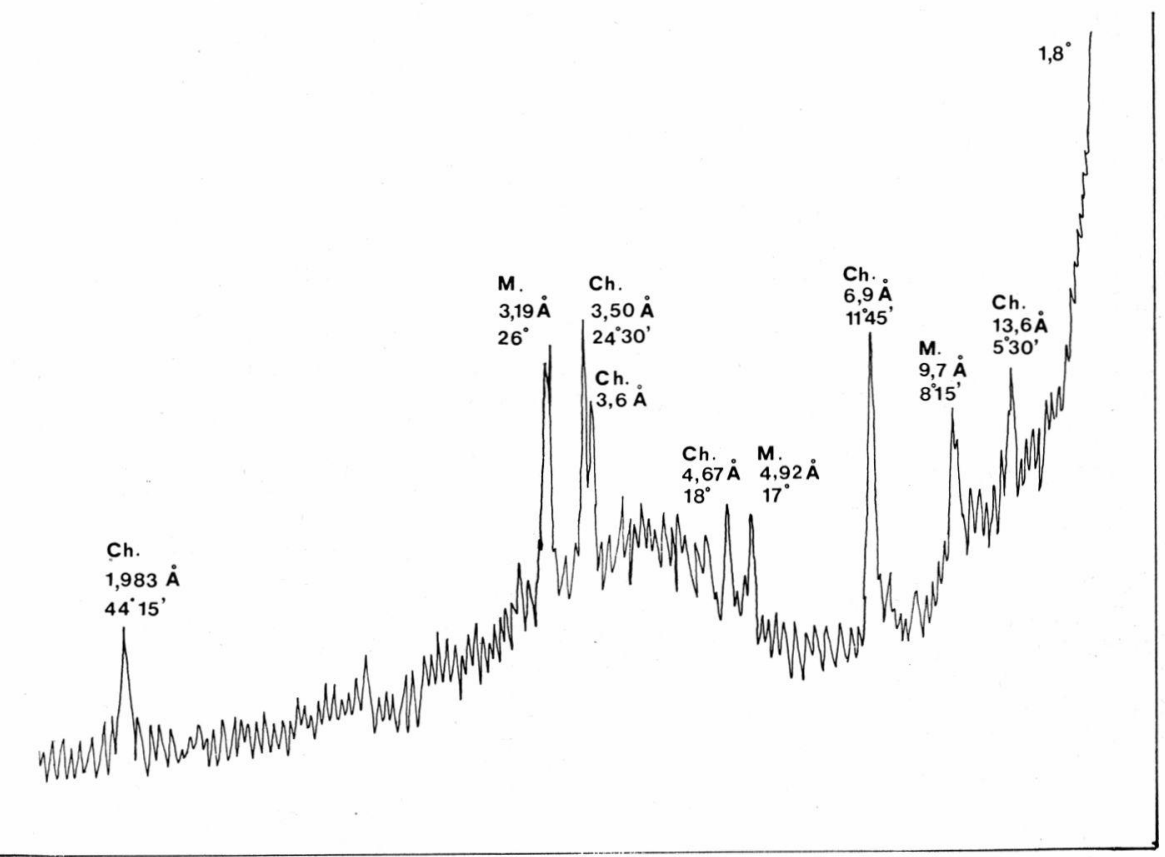

FIG. 5. - Diagramme aux rayons $\mathrm{X}$ des grains inféricurs à 2 microns. 
Les mesures effectuées, à des hauteurs différentes, dans des fragments de carottes montrent une assez grande homogénéité. Les diagrammes de rayons $\mathrm{X}$ des grains inférieurs à 2 microns, effectués sur plaquettes orientées, sont identiques ; les raies principales (fig. 5) sont celles des produits d'altération des silicates de la granodiorite : micas très fins, biotite, séricite et chlorite. Ces minéraux sont peu altérés : après traitement au glycol aucun gonflement n'apparaît.

La détermination de ces minéraux a été précisée par l'analyse thermique différentielle et par l'analyse des surface spécifiques.

Cette dernière méthode d'analyse montre que les valeurs des surfaces spécifiques croissent de bas en haut (fig. 6). Elles reflètent et confirment la diminution de la taille des grains ; elles peuvent également traduire une augmentation de la proportion de chlorite vers la surface. Après lessivage à l'acide chlorhydrique $0,1 \mathrm{~N}$ les valeurs des surfaces spécifiques restent sensiblement les mêmes, montrant ainsi l'obturation encore solide des feuillets des phyllites. Dans les échantillons B et C cependant, l'écart entre les mesures est notable.

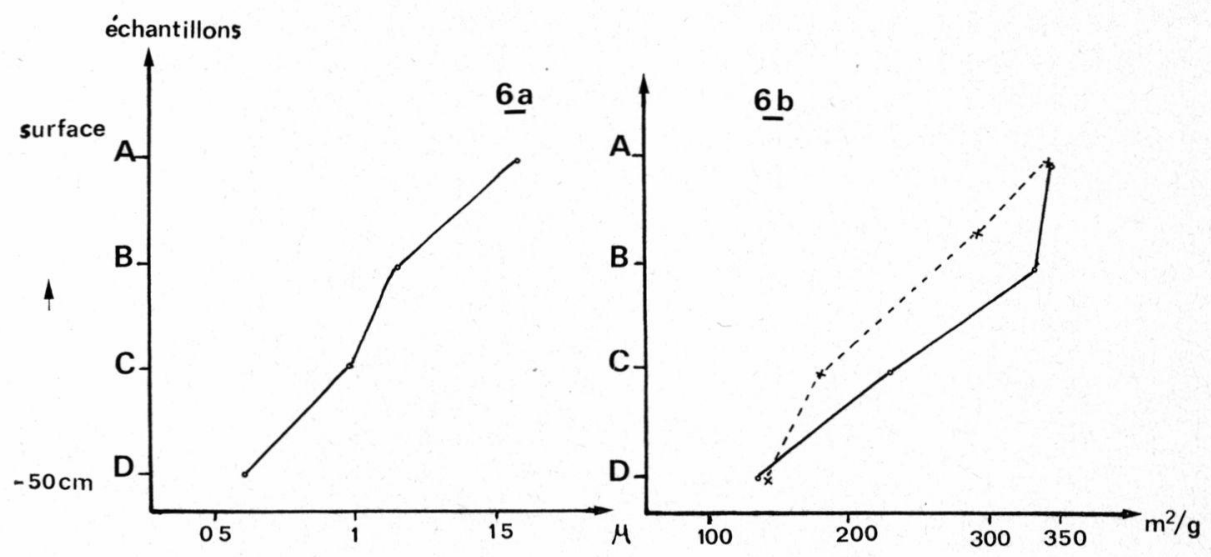

FIG. 6. - Variations verticales de la médiane (6 a) comparée à la variation verticale des surfaces spécifiques $(6 \mathrm{~b})$; —- échantillon traité par $\mathrm{HCl}$; -..- échantillon normal.

Si la finesse de grain des sédiments est très grande, exceptionnelle même, il apparaît que la vitesse d'altération de la roche-mère (granodiorite) est faible ; la dégradation des minéraux est, ellemême, très lente et ceci résulte vraisemblablement des conditions de milieu : températures toujours basses, faible agressivité de l'eau ...

Du fait que l'on ne connaît pas le taux de sédimentation et compte tenu des perturbations structurales subies par le sédiment, il est difficile d'avoir une idée exacte de cette vitesse d'altération. 


\section{3. - ANALYSE CHIMIQUE DES SEDIMENTS}

\section{1. - Méthodes.}

Les échantillons de sédiment, prélevés tous les 3 à $4 \mathrm{~cm}$ sur une carotte desséchée à l'air libre, sont réduits en poudre dans un mortier. Toutes les analyses sont effectuées à partir de ce sédiment sec et broyé. Les résultats sont exprimés par rapport au poids sec, déterminé après passage à l'étuve à $110^{\circ}$ pendant une nuit.

- La matière organique est estimée gravimétriquement après ignition dans un four à moufle. Les échantillons sont calcinés à $550^{\circ}$ pendant deux heures comme le conseille Ungemach (1960 in Rybak 1969). Selon ces auteurs et dans de telles conditions, seulement $0,4 \%$ des carbonates sont détruits; dans les sédiments considérés les carbonates sont à l'état de traces ; il n'était donc pas nécessaire de les régénérer.

- Parallèlement nous avons déterminé, par conductibilité thermique dans un four à carbone Leco, la teneur en carbone organique ; l'azote a été dosé au four Coleman (dosage volumétrique de $\mathrm{N}$ après élimination du $\mathrm{CO}^{2}$ vecteur).

- Les dosages des principaux cations : Ca, Mg, Na, K, Fe, Mn, ont été effectués sur des solutions préparées selon la méthode de Mackereth (1963 et 1966) légèrement modifiée. $0,25 \mathrm{~g}$ de sédiment sec sont attaqués à chaud par $10 \mathrm{ml}$ d'acide fluorhydrique à $40 \%$, $3 \mathrm{ml}$ d'acide nitrique et $3 \mathrm{ml}$ d'acide perchlorique dans des béchers en polypropylène (Soreflon). Après évaporation, le résidu est dissous dans $10 \mathrm{ml}$ d'HCl $2 \mathrm{M}$ et ramené à $100 \mathrm{ml}$. Les analyses des divers cations sont réalisées sur des fractions aliquotes de cette solution, à l'aide d'un spectrophotomètre Unicam SP. 90.

\section{2. - Matière organique, carbone, azote.}

Les teneurs en matière organique, comprises entre 19 et $24 \%$ du poids sec, sont relativement élevées. Elles croissent régulièrement de $-25 \mathrm{~cm}$ vers la surface et la teneur maximale s'observe au contact vase - eau (fig. 7 ).

Selon Mackereth (1966) les principaux facteurs influençant la composition des sédiments lacustres sont essentiellement externes. Ce sont :

- la variation du taux de dépôt de matière organique en fonction des changements de productivité du bassin versant ;

-- la variation du taux de dépôt de matière minérale qui dépend de l'intensité de l'érosion sur le bassin versant ; 
-.- la variation dans la vitesse de minéralisation de la matière organique à la surface du sédiment; ce dernier facteur, apparemment interne, est soumis aux conditions climatiques externes.

Les valeurs élevées que nous obtenons sont difficilement explicables compte tenu de l'aridité actuelle du bassin versant et du caractère oligotrophe de la masse d'eau. Ces valeurs s'expliquent certainement par les conditions de milieu (température faible, déficit en $\mathrm{O}^{2}$ ) qui ralentissent les processus de minéralisation (Laville $1971 \mathrm{~b})$.

Les teneurs en carbone organique sont de l'ordre de 8,5 à 9,5\% et les teneurs en azote total sont voisines de $1 \%$ (tableau I). Le rapport $\mathrm{C} / \mathrm{N}$ est constant tout au long de la colonne de sédiment analysé. Ceci, ajouté à la faible valeur de ce rapport (voisin de 9), traduit le faible taux de minéralisation évoqué plus haut.

TABleau I. - Analyse du carbone, de l'azote et valeurs du rapport $\mathrm{C} / \mathrm{N}$ à quatre profondeurs différentes dans le sédiment.

\begin{tabular}{rccc}
\hline Profondeur en cm & C en $\%$ & N en $\%$ & C/N \\
\hline 5 & 9,22 & 1,02 & 9,04 \\
12 & 8,89 & 0,97 & 9,16 \\
30 & 9,77 & 1,04 & 9,39 \\
40 & 8,55 & 0,92 & 9,29 \\
\hline
\end{tabular}

Si l'on se réfère aux données de Rybak (1969) concernant les lacs polonais, les sédiments considérés seraient des dépôts d'un lac de type mésotrophe (matière organique : 17 à $29 \%$ avec une

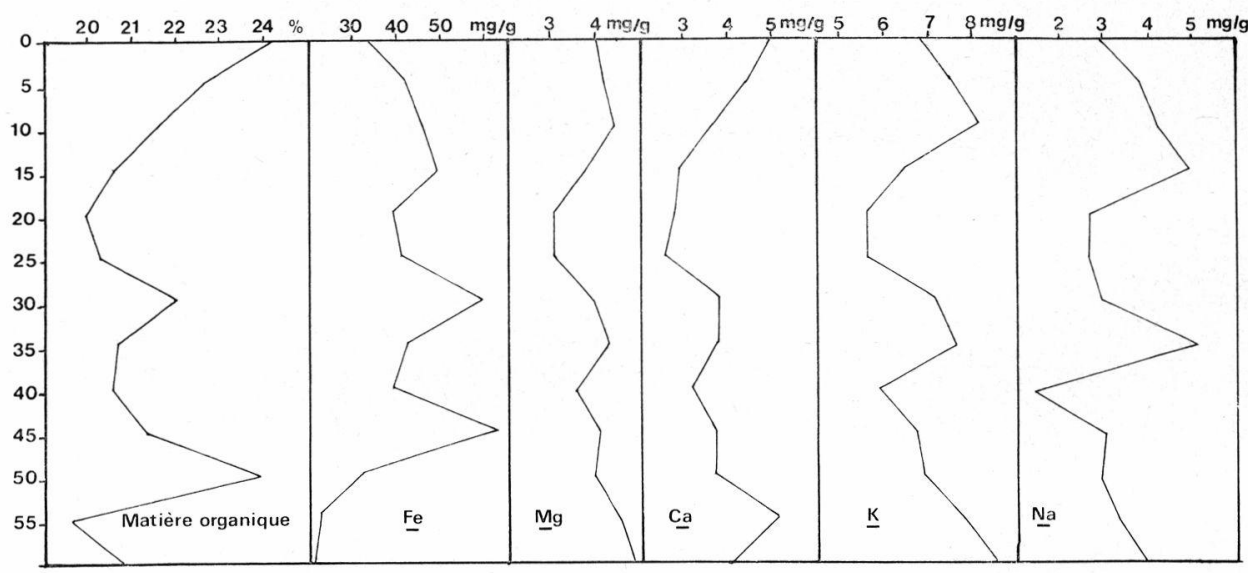

Fig. 7. - Distribution verticale des teneurs en matière organique, fer, calcium, magnésium, sodium et potassium. 
moyenne de $22,67 \%$ ). Mais les teneurs en calcium et le rapport $\mathrm{C} / \mathrm{N}$ sont éloignés des valeurs habituellement rencontrées dans cette catégorie de lac.

On pourrait y voir toutefois une preuve de l'origine exogène de la matière organique enfouie dans les sédiments.

\section{3. - Les cations.}

\subsection{1. - Fer ET MANGANĖSE.}

Dans la nature le fer se présente soit à l'état minéral (sels ferreux et sels ferriques) soit à l'état organique (collö̈des, humates). Dans la colonne de sédiment analysé, à l'exception de la couche superficielle, les teneurs en fer varient parallèlement à celles en matière organique (fig. 7 ). Le fer semble done essentiellement lié à la fraction organique du sédiment. Cette relation semble normale vu le rôle important que joue le fer dans le métabolisme des plantes et des animaux (Hem 1970). D'autre part la fraction non assimilable du fer (Rhode 1948 in Dussart 1966) se trouve principalement sous forme de composés organiques, de colloïdes, de complexes humiques à grande capacité d'adsorption. Les teneurs en manganèse sont faibles; clles varient de $1,1 \mathrm{mg} / \mathrm{g}$ à $1,5 \mathrm{mg} / \mathrm{g}$. Les teneurs en $\mathrm{Mn}$ et $\mathrm{Fe}$ sont voisines des valeurs moyennes fournies par Hem (1970) pour les roches éruptives; $0,94 \mathrm{mg} / \mathrm{g}$ de $\mathrm{Mn}$ et $42,2 \mathrm{mg} / \mathrm{g}$ de $\mathrm{Fe}$. Les apports en $\mathrm{Fe}$ non organique et en Mn se feraient donc surtout sous forme détritique.

Le lessivage du bassin versant ne joue qu'un rôle secondaire car les formes oxydées de ces deux éléments ne présentent qu'un faible taux de solubilité (Mackereth 1966). Au scin du lac, l'alternance entre une phase réductrice et une phase oxydante provoque une redissolution du fer à partir des sédiments ou la précipitation des sels ferriques. Ce phénomène a déjà été mis en évidence dans ce lac par Capblancq et Laville (1968) et Cablancq (1972) lors d'analyses régulières de l'eau.

\subsection{2. — Ca, Mg, K, Na.}

Ces cations sont généralement associés à la fraction minérale. La courbe de répartition verticale des métaux alcalino-terreux dans le sédiment, $\mathrm{Mg}$ notamment, est identique à celle des métaux alcalins (fig. 7 ). Les teneurs en calcium et en magnésium du sédiment sont très voisines. Le rapport $\mathrm{Mg} / \mathrm{Ca}$ varie de 0,8 à 1,3 . Dans l'eau, par contre, les teneurs en Ca sont nettement supérieures à celles en $\mathbf{M g}$ et le rapport $\mathrm{Mg} / \mathrm{Ca}$ est de 0,07 à 0,17 (Capblancq et Laville 1968). 
Ceci traduit la solubilité différentielle de ces cations : Mg est moins facilement lessivé du sol que ne l'est le Ca.

Le même phénomène s'observe pour le sodium et le potassium. Dans l'eau, le potassium est moins abondant que le sodium. Dans les sédiments, il y a plus de potassium que de sodium. Le $\mathbf{K}$ étant légèrement moins abondant que le $\mathrm{Na}$ dans les roches éruptives, les teneurs observées s'expliquent par les différences essentielles de solubilité entre ces deux métaux alcalins :

--- le sodium, après libération, tend à rester en solution ;

- le potassium, plus difficilement libéré par les minéraux silicatés, montre une forte tendance à être incorporé dans les produits de dégradation et notamment les argiles.

La composition chimique des sédiments ne reflète pas exactement la nature chimique du bassin versant. Les éléments analysés sont nettement moins abondants dans les sédiments que dans les roches éruptives (concentrations moyennes dans les roches éruptives d'après Hem (1970) : Ca : $36,2 \mathrm{mg} / \mathrm{g} ; \mathrm{Na}: 28,1 \mathrm{mg} / \mathrm{g}$; K : $25,7 \mathrm{mg} / \mathrm{g} ; \mathrm{Mg}: 17,6 \mathrm{mg} / \mathrm{g})$. Selon l'hypothèse de Mackereth (1966) ceci laisse présager que ces sédiments ne dérivent pas directement de l'érosion mécanique du bassin versant mais semblent plutôt refléter le comportement des ions vis-à-vis de l'eau — lessivage, redissolution à partir des sédiments — et la stabilité de leurs solutions. Ces phénomènes (lessivage notamment) semblent donc jouer un rôle plus important dans la formation des sédiments de la zone profonde du lac que l'érosion mécanique. Ceci traduirait donc l'absence d'apports détritiques importants dans cette zone (nous avons vu qu'il n'y avait pas de ruisseau «affluent » donc pas d'arrivée de charge solide). Rappelons que le lessivage s'effectue toujours avec une perte nolable par l'émissaire.

L'analyse des sédiments de la zone littorale, qui sont sous la dépendance des apports directs au niveau des berges, permettra de préciser l'origine des sédiments du lac de Port-Bielh. L'étude sur des caroltes plus longues de la répartition des divers éléments devrait également, à la lumière de la paléoclimatologie, confirmer les hypothèses émises et nous permettre de retracer l'histoire de ce lac de montagne.

\section{4. - REPARTITION VERTICALE DE LA FAUNE DANS LE SEDDIMENT}

Parallèlement à l'étude sédimentaire, il nous a paru intéressant d'étudier la répartition verticale de la faune dans le substrat de la zone profonde du lac de Port-Biclh. Une telle répartition a déjà été envisagée par de nombreux auteurs et notamment par Berg 
(1938), Cole (1953), Kajak et Dusoge (1971), Lenz (1931), Milbrink (1969). Ces divers travaux démontrent que la majorité de la faune vit dans la couche superficielle du sédiment. Cependant, les Oligochètes et certaines larves de Diptères peuvent pénétrer assez profondément dans le substrat.

\section{1. - Résultats.}

Pour la série de prélèvements du 11-11-1973, nous avons examiné séparément l'eau surnageante et la première couche de vase; le 26-11-1973 nous les avons assimilées. Les résultats obtenus sont consignés dans les tableaux II et III.

Tableau II. - Répartition verticale de la faune dans le sédiment (26-11-1973).

Hauteur en $\mathrm{cm}$

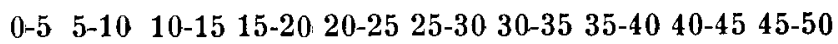

\begin{tabular}{|c|c|c|c|c|c|c|c|c|c|c|}
\hline Nématodes & 4 & 1 & & & & & & & & \\
\hline Planaires & 15 & 1 & & & & & & & & \\
\hline Glossiphonia complanata & 1 & & & & & & & & & \\
\hline Tubifex tubifex & 27 & 25 & 10 & 9 & 10 & 9 & 4 & 2 & 1 & 2 \\
\hline Peloscolex pyrenaicus & 71 & 22 & 8 & & 3 & 2 & & 1 & & \\
\hline Cocons d'oligochètes & 1 & 2 & & & & & & & & \\
\hline Cypria lacustris & 276 & 9 & 4 & 1 & 1 & & & & & \\
\hline Canthocamptus staphylinu & $s \quad 42$ & 2 & 1 & 1 & & & & & & \\
\hline Cyclopides divers & 4 & & & & & & & & & \\
\hline Zavrelimyia melanura & 4 & & 1 & 1 & & & & & & \\
\hline Psectrocladius sordidellus & 1 & 1 & 1 & & & & & & & \\
\hline Chironomus plumosus & 18 & 9 & 4 & 2 & & & & & & \\
\hline Tanytarsini & 81 & 12 & 1 & 1 & & & & . & & \\
\hline Sialis lutaria & 3 & & & & & & & & & \\
\hline Total & 548 & 84 & 30 & 15 & 14 & 11 & 4 & 3 & 1 & 2 \\
\hline$\%$ & 76,9 & 11,78 & 4,2 & 2,1 & 1,9 & 1,5 & 0,56 & 0,42 & 0,14 & 0,28 \\
\hline
\end{tabular}

Il apparaît que $76,9 \%$ de la faune vit dans les 5 premiers centimètres de sédiment et que près de $90 \%$ se trouve concentrée dans les 10 centimètres supérieurs. Dans une carotte de $15 \mathrm{~cm}$ de hauteur on est donc assuré de recueillir 92 à $96 \%$ du benthos, à condition de conserver l'eau surnageante qui abrite $8,5 \%$ du peuplement. Il faut cependant noter que les divers groupes systématiques se répartissent différemment dans l'épaisseur du substrat. 
TABlEaU III. - Répartition verticale de la faune dans le sédiment (11-11-1973).

\begin{tabular}{|c|c|c|c|c|c|c|c|}
\hline Hauteur en $\mathrm{cm}$ & 5 & 10 & 15 & 25 & 35 & 45 & $\mathrm{H}_{2} \mathrm{O}$ \\
\hline Psectrocladius sordidellus & 1 & & & & & & 2 \\
\hline Chironomus plumosus & 18 & 19 & 2 & & & & \\
\hline Zavrelimyia melanura & 3 & & & & & & 6 \\
\hline Tanytarsini & 130 & 22 & 1 & & & & 2 \\
\hline Tubifex tubifex & 40 & 70 & 18 & 7 & $\mathbf{5}$ & & \\
\hline Peloscolex pyrenaicus & 64 & 26 & 2 & 2 & & 1 & \\
\hline Cocons & 1 & 3 & 1 & & & & \\
\hline Lumbriculus variegatus & 1 & & & & & & \\
\hline Cypria lacustris & 349 & 50 & 3 & 1 & & & 21 \\
\hline Canthocamplus staphylinus & 84 & 11 & & & & & 21 \\
\hline Candona neglecta & 6 & 1 & & & & & \\
\hline Cladocères & 31 & 1 & & & & & 28 \\
\hline Glossiphonia complanata & 1 & & & & & & \\
\hline Planaires & 9 & 1 & 1 & & & & 12 \\
\hline Nematodes & 3 & & & & $\cdots$ & & \\
\hline Cyclopides & 1 & 1 & & & & & 6 \\
\hline Total : 1084 & 742 & 205 & 28 & 10 & 5 & 1 & 93 \\
\hline$\%$ & 68,4 & 18,9 & 2,5 & 0,9 & 0,45 & 0,09 & 8,5 \\
\hline
\end{tabular}

\subsection{1. — Les Chironomides.}

Les données concernant ce groupe sont résumées dans le tableau IV. Ces Diptères ont une abondance maximale $(75,9 \%)$ dans les $5 \mathrm{~cm}$ supérieurs, et $92 \%$ vivent dans les $10 \mathrm{~cm}$ supérieurs ; ceci est en accord avec les résultats de Milbrinck (1969) et de Kajak (1958 in Kajak et Dusoge 1971) pour les lacs et de Ford (1962) pour les eaux courantes. Dans les sédiments que nous avons examinés ils ne pénètrent pas au-delà de $20 \mathrm{~cm}$. On trouve dans la littérature quelques observations au-delà de cette profondeur : Digerfeldt et Lettewal (1969) signalent notamment quelques rares individus à $50 \mathrm{~cm}$ sous l'interface vase - eau. Nous noterons également que les 5 premiers centimètres de vase hébergent $85 \%$ des Tanytarsini contre seulement $54,5 \%$ des Chironomus plumosus. Entre 10 et $15 \mathrm{~cm}$ et entre 15 et $20 \mathrm{~cm}$ nous trouvons encore respectivement 12,1 et $6 \%$ des Chironomus plumosus contre seulement $1 \%$ des Tanytarsini. Il est bien connu que les gros spécimens de Chironominae pénètrent assez profondément dans le substrat : $12 \mathrm{~cm}$ (Efford 1960), $22 \mathrm{~cm}$ (Lenz 1931), $35-40 \mathrm{~cm}$ dans les sédiments mous et peu minéralisés du lac Esrom (Berg 1938). Chironomus plumosus se rencontre jusqu'à des profondeurs variables : 
$8 \mathrm{~cm}$ (Kajak 1958), $12 \mathrm{~cm}$ (Cole 1953), $20 \mathrm{~cm}$ (Milbrink 1969). Nous avons récolté cette espèce jusqu'à $20 \mathrm{~cm}$ dans les sédiments du lac de Port-Bielh.

Tableau IV. - Répartition verticale des Chironomides dans le sédiment.

\begin{tabular}{lccccr}
\hline & $0-5 \mathrm{~cm}$ & $5-10 \mathrm{~cm}$ & $10-15 \mathrm{~cm}$ & $15-20 \mathrm{~cm}$ & To- \\
\hline Zavrelimyia melanura & 4 & 1 & 1 & & 6 \\
Psectrocladius sordidellus & 1 & 1 & 1 & & 3 \\
Chironomus plumosus & $18(54,5 \%)$ & $9(27,2 \%)$ & $4(12,1 \%)$ & $2(6 \%)$ & 33 \\
Tanytarsini & $81(85,2 \%)$ & $12(12,6 \%)$ & $1(1 \%)$ & $1(1 \%)$ & 95 \\
Total & 104 & 23 & 7 & 3 & 137 \\
$\%$ & 75,9 & 16,8 & 5,1 & 2,2 & \\
\hline
\end{tabular}

D'après Berg (1938), Cole (1953), Lenz (1931), Särkkä et Paasivirta (1972), l'abondance maximum des Tanypodinae se situe entre 5 et $8 \mathrm{~cm}$, ils sont encore présents à $14-15 \mathrm{~cm}$ (Efford 1960, Lenz 1931). Nos observations diffèrent légèrement : nous avons récolté Zavrelimyia melanura essentiellement dans la couche la plus superficielle et exceptionnellement jusqu'à $15 \mathrm{~cm}$. Cette espèce, comme beaucoup de Tanypodinae, est une forme nageuse et elle peut évoluer dans l'eau sus-jacente (tableau III).

\subsection{2. - LES Oligochìtes.}

Ils pénètrent plus profondément que les Chironomides à l'intérieur du sédiment (tableau V). Si 47,5\% vivent dans les 5 premiers centimètres et $75,1 \%$ dans les $10 \mathrm{~cm}$ supérieurs, on en trouve jusqu'à des profondeurs pouvant atteindre $45-50 \mathrm{~cm}$. Notons que si l'abondance maximale se situe toujours dans les premiers centimètres - parfois légèrement en dessous de l'interface vase - eau - les profondeurs atteintes sont nettement supérieures à celles mentionnées dans la littérature : $30 \mathrm{~cm}$ (Berg 1938), $18-24 \mathrm{~cm}$ (Lenz 1931), $10-11 \mathrm{~cm}$ (Brinkhurst et Kennedy 1965 ), $20 \mathrm{~cm}$ Brinkhurst et al. 1969), $14-16 \mathrm{~cm}$ (Efford 1960, Särkkä et Paasivirta 1972). Les deux seules espèces de Tubificidae vivant dans la zone profonde présentent la même abondance et atteignent des profondeurs maximales identiques. Cependant Peloscolex pyrenaicus semble vivre plus en surface - 86,8\% dans les $10 \mathrm{~cm}$ superficiels - que Tubifex tubifex qui est abondant dans la zone de 0 à $30 \mathrm{~cm}(90 \%)$; Sapkarev (1959 in Brinkhurst et Jamieson 1971) avaient déjà noté une telle subdivision du sédiment en zones préférentielles pour les diverses espèces d'Oligo- 
chètes ; mais Brinkhurst et Kennedy (1965) n'ont pas retrouvé cette zonation.

Kajak et Dusoge (1971) ont mis en évidence une relation entre la taille des organismes, notamment les Chironomides, et leur position dans le substrat. C'est ainsi que la taille des représentants de chaque espèce croît avec la profondeur, les stades jeunes étant concentrés à la surface. Dans le milieu marin, Birkett (1958) a montré que les animaux les plus âgés pénétraient plus profondément que les juvéniles. Coleman et Hynes (1970), dans un cours d'eau n'ont pas retrouvé cette corrélation. Afin de rechercher une

Tableau VI. - Amplitude et moyenne de la variation de la largeur du $10^{\mathrm{e}}$ segment de Tubifex tubifex en fonction de la profondeur.

\begin{tabular}{ccc}
\hline Profondeur en cm & Amplitude $(\mathrm{mm})$ & Moyenne $(\mathrm{mm})$ \\
\hline $0-5$ & $0,15-0,75$ & 0,4 \\
$5-10$ & $0,2-0,75$ & 0,5 \\
$10-15$ & $0,3-0,75$ & 0,6 \\
$20-25$ & $0,5-0,75$ & 0,66 \\
$25-30$ & $0,6-0,75$ & 0,69 \\
\hline
\end{tabular}

telle relation nous avons mesuré la largeur du dixième segment des Tubifex tubifex que nous avions prélevés (tableau VI). Nous avons choisi ce critère car la longueur du corps présente dans ce groupe des variations importantes en fonction de la plus ou moins grande contraction de l'animal. Il apparaît une relation entre la taille moyenne de l'animal et sa situation dans le sédiment : la taille moyenne croît lorsque la profondeur augmente.

\subsection{3. - Groupes DIVErS.}

Les Crustacés, groupe numériquement abondant dans le benthos, sont concentrés $(89-95 \%)$ dans le premier niveau des sédiments $(0-5 \mathrm{~cm})$ et la quasi totalité vit dans les $10 \mathrm{~cm}$ supérieurs (tableaux VII et VIII). Seul l'Ostracode Cypria lacustris peuple faiblement mais de façon régulière la zone comprise entre 5 et $15 \mathrm{~cm}$. Efford (1960) a trouvé des Ostracodes jusqu'à $12,5 \mathrm{~cm}$ de profondeur dans le lit d'un cours d'eau. Les Copépodes et les Cladocères vivent dans la strate superficielle du sédiment et nous en avons recueillis dans la couche d'eau sus-jacente (tableau III). Cette répartition générale des Crustacés est en accord avec les travaux de Hakala (1971), Milbrink (1969) et Särkkä et Paasivirta (1972). 
Tableau VII. - Répartition verticale des Crustacés dans le sédiment le 11-11-1973.

\begin{tabular}{lrrccc}
\hline Profondeur en cm & $0-5$ & $5-10$ & $10-15$ & $15-25$ & H 20 \\
\hline Cypria lacustris & 349 & 50 & 3 & 1 & $\mathbf{2 1}$ \\
Candona neglecta & $\mathbf{6}$ & $\mathbf{1}$ & 1 & & $\mathbf{2 1}$ \\
Canthocamptus staphylinus & $\mathbf{8 4}$ & $\mathbf{1 1}$ & & & $\mathbf{6}$ \\
Cyclopides divers & 1 & 1 & & & $\mathbf{2 3}$ \\
Cladocères & 31 & 1 & & & $\mathbf{7 1}$ \\
Total & 471 & $\mathbf{6 4}$ & 4 & 1 & 11,6 \\
$\%$ & 77 & $\mathbf{1 0 , 3}$ & 0,6 & 1 & \\
\hline
\end{tabular}

Tableav VIII. - Répartition verticale des Crustacés dans le sédiment le 26-11-1973.

\begin{tabular}{lrcccc}
\hline Profondeur en cm & $0-5$ & $5-10$ & $10-15$ & $15-20$ & $20-25$ \\
\hline Cypria lacustris & 276 & 9 & 4 & 1 & 1 \\
Canthocamptus staphylinus & 42 & 2 & 1 & 1 & \\
Cyclopides divers & 4 & & & & \\
Total & 322 & 11 & 5 & 2 & 1 \\
$\%$ & 94,4 & 3,2 & 1,4 & 0,5 & 0,25 \\
\hline
\end{tabular}

Le faible nombre de données concernant les autres groupes ne nous permet pas de tirer de conclusion générale sur leur distribution verticale. Les Planaires semblent toutefois préférer la zone de contact entre la vase et l'eau et nous les avons essentiellement récoltées dans l'eau surnageante (tableau III). Sialis lutaria est peu abondant à cette profondeur dans le lac de Port-Bielh (Giani et Laville 1973) mais les rares exemplaires capturés occupaient la pellicule de vase supérieure (tableau III) alors que Ford (1962) et Pugh (1959) en avaient trouvé jusqu’à $15 \mathrm{~cm}$ dans le sédiment.

\section{2. - Discussion.}

La confrontation de nos résultats avec ceux des divers auteurs cités montre une certaine régularité dans la répartition verticale du benthos et les preferendums des divers groupes. Malgré cette régularité dans la répartition verticale quantitative, déjà signalée par Kajak et Dusoge (1971), il semble toutefois que la pénétration de la faune à l'intérieur du substrat soit plus importante dans les sédiments que nous avons considérés. Ceci peut être mis en relation avec la structure même du fond du lac. En effet l'étude des sédiments montre que la vase se caractérise par sa faible densité et sa granulométrie où dominent les fractions fines $(50 \mu)$. Les deux premières couches de sédiment ( 3 à $5 \mathrm{~cm}$ et 5 à $25 \mathrm{~cm}$, cf. 2.1.), formées de sédiments très fluides, abritent $94,7 \%$ de la faune. 
Dans la zone suivante $(25-50 \mathrm{~cm})$, plus compacte, on ne rencontre que $5 \%$ de la faune. Enfin les cinq derniers centimètres de la carotte sont constitués par une couche grise, assez compacte où l'on ne recueille que $0,2 \%$ du peuplement total. Ford (1962) avait déjà insisté sur le rôle de la structure du sédiment — matière organique, granulométric - et notamment de la densité sur la distribution verticale de la faune. Nous avons vu que la densité du sédiment, très faible, est homogène dans les trois premières couches, c'est-à-dire de 0 à $45-50 \mathrm{~cm}$ et qu'elle croît légèrement au niveau de la quatrième couche. La faible compacité du sédiment et l'homogénéité de cette densité permet certainement la pénétration de la faune dans les 45 -50 premiers centimètres jusqu’à la strate plus compactée.

Pugh (1959) accorde peu d'importance à la granulométrie dans la détermination de la répartition verticale dans le sédiment. Il souligne plus particulièrement le rôle de la morphologie des espèces ainsi que celui de leur physiologie : la présence d'un pigment respiratoire (Tubificidae, Chironomes) favorise la pénétration dans les zones à faible teneur en $\mathrm{O}^{2}$.

Les organismes détritivores, tels les Tubificidae et certains Chironomides, trouvent dans des sédiments aussi peu minéralisés de la nourriture jusqu'à des profondeurs assez importantes ; ils restent cependant tributaires - comme l'avaient signalé Brinkhurst et al. (1969), Brundin (1950), Kajak et Dusoge (1971) — de l'oxygène qu’ils doivent aller prélever près de l'interface vase-eau. Brundin (1950) a montré que lorsque la teneur en $\mathrm{O}^{2}$ est forte les besoins en oxygène sont rapidement satisfaits ce qui permet à l'animal, entre deux phases respiratoires, de pénétrer à l'intérieur du sédiment pour se nourrir, or nos prélèvements ont été réalisés juste après la circulation d'automne, alors que l'eau du fond est riche en $\mathrm{O}^{2}$.

Si la teneur en $\mathrm{O}^{2}$ détermine le lemps de pénétration, el si la compacité régit la possibilité de fouissement, la profondeur de pénétration est sous la dépendance combinée de ces deux facteurs mais aussi du mode de nutrition ; seuls les détritivores pénètrent généralement dans le sédiment. Sachant que certains groupes vivent essentiellement à la surface et que les autres sont obligés de venir y respirer, la densité du peuplement est toujours maximale à la surface (Brinkhurst et Kennedy 1965) ; c'est là que se trouvent les prédateurs, formes d'ailleurs généralement nageuses : Zavrelymia melanura, Sialis lutaria.

Kajak et Dusoge (1971) ont également mis en évidence le rôle de la pression de prédation des poissons qui déterminerait un certain enfoncement de la faune et notamment des Oligochètes dans le substrat. Nous n'avons que peu de données concernant le peu- 
plement en truites (Salmo trutta fario L.) du lac de Port-Bielh et il ne nous est pas possible de tirer des conclusions sur leur rôle. Brinkhurst et Kennedy (1965) ont noté un phénomène de compétition entre les espèces du genre Chironomus et certains Tubificidae : lorsque le nombre de Chironomus croît les Tubificidae régressent. Cette compétition ne semble pas exister dans le lac de PortBielh entre Chironomus plumosus et les deux espèces de Tubificidae (Tubifex tubifex et Peloscolex pyrenaicus) car les deux Tubificidae atteignent leur abondance maximum dans la zone où vit le Chironomide.

Les mouvements incessants de la faune à travers le sédiment affectent la structure de celui-ci. Kleckner (1967 in Stockner et Lund 1970) et Stockner et Lund (1970) ont d'ailleurs analysé cette action des organismes qui peut se résumer comme suit :

- fouissement puis remplissage du trou par des sédiments venus àau-dessus ;

- construction de tubes avec transports de matériaux ;

- transport passif par ingestion de sédiments à un niveau et rejet à un niveau différent (Oligochètes);

- transport de matériel agglutiné sur le corps (soies d'Oligochètes, mucus ...).

Cette action des organismes est surtout importante dans les $10 \mathrm{~cm}$ supérieurs qui sont les plus densément peuplés. Dans le lac de Port-Bielh, les Oligochètes peuvent remanier le sédiment jusqu'à des profondeurs de l'ordre de $40-50 \mathrm{~cm}$. Compte tenu de la très faible vitesse de sédimentation dans ce lac, cetle action des organismes sur la stratification des sédiments est très importante. Mais elle ne suffit vraisemblablement pas pour expliquer le granoclassement que nous avons constaté. 


\section{TRAVAUX CITÉS}

BERg (K.). 1938. - - Studies on the bottom animals of Esrom Lakes. K. Vidensk. Selsk. Naturv. Math. Avd., 9 : 1-255.

Birketr (L.). 1958. - A basis for comparing grabs. J. Cons. Perm. Int. Explor. Mer., 23 : 202-207.

Brinkhurst (R. O.), Chua (K. E.) et Batoosingh (E.). 1969. - Modifications in sampling procedures as applied to studies on the bacteria and tubificid Oligochaetes inhabiting aquatic sediments. J. Fish. Res. Bd. Can., $26: 2581-2593$.

Brinkhurst (R. O.) et Jimieson (B.G.M.). 1971. - Aquatic Oligochaeta of the World. Oliver and Boyd. Edinburgh. $860 \mathrm{p}$.

Brinkhurst (R. O.) et Kennedy (C. R.). 1965. - Studies on the biology of the Tubificidae (Annelida, Oligochaeta) in a polluted stream. J. Anim. Ecol., 34: 429-443.

BRUNDin (L.). 1950. - The relation of $\mathrm{O}_{2}$-microstratification at the mud surface to the ecology of the profundal bottom fauna. Rep. Inst. Fresh. Res. Drott., 31 : 32-42.

Bücher (A.) et I.uCAS (C.). 1972. - Le nuage de poussière rouge du 7 février 1972. Bull. Soc. Hist. Nat. Toulouse, $108:$ 437-445.

Capblance (J.), 1972. - Phytoplancton et productivité primaire de quelques lacs d'altitude dans les Pyrénées. Annls Limnol., 8 : 231-321.

Capblance (J.). 1973. - Phytobenthos et productivité primaire d'un lac de haute montagne dans les Pyrénées centrales. Annls limnol., $9: 193-230$.

Capblance (J.) et Laville (H.). 1968. - Etude morphométrique et physico-chimique de neuf lacs du massif de Néouvielle (HautesPyrénées). Annls limnol., 4 : 275-324.

Cole (G. A.). 1953. - Notes on the vertical distribution of organisms in the profundal sediments of Douglas Lake, Michigan. Amer. Midl. Nat., $49: 252-256$.

Coleman (M. J.) et Hynes (H.B.N.). 1970. - The vertical distribution of the invertebrate fauna in the bed of a stream. Limnol. Oceanog., $15: 31-40$.

Digerfeldi (G.) et Lettevall (V.). 1969. - A new type of sediment sampler. Geologiska, Föreningens Stockholm Förhandlingar, 91 : 399-406.

Dussart (B.). 1966. - Limnologie. Etude des equx continentales. Gauthier-Villars ed., Paris. 1966. 677 p.

EFrord (II. E.). 1960. - A method of studying the vertical distribution of the bottom fauna. Hydrobiol., $16: 288-292$.

ForD (J. B.). 1962. - The vertical distribution of larval Chironomidae (Dipt.) in the mud of a stream. Hydrobiol., $19: 262-272$.

GIANI (N.). 1974. - Description d'un nouveau type de carottier pour les sédiments très fluides. Annls limnol., $10: 99-108$.

Gian. (N.) et Laville (H.). 1973. - Cycle biologique et production de Sialis lutaria L. (Megaloptera) dans le lac de Port-Bielh (Pyrénées centrales). Annls limnol., 9 : 45-61. 
Hakata (I.). 1971. - A new model of the Kajak bottom-sampler and other improvements in the zoobenthos sampling technique. Ann. Zool. Fenn., $8: 422-426$.

HEM (J. D.). 1970. - Study and interpretation of the chemical caracteristics of natural water. $2^{e}$ éd. 1971. Geological survey watersupply paper 1473 . United states Government Printing Office, Washington : $363 \mathrm{p}$.

Juget (J.) et Giani (N.). 1974. - Répartition des Oligochètes lacustres du massif de Néouvielle (Hautes-Pyrénées) avec la description de Peloscolex pyrenaicus, n, sp. Annls limnol., 10 : 33-53.

KaJAK (Z.) et Dusoge (K.). 1971. - The regularities of vertical distribution of benthos in bottom sediments of three Masurian Lakes. Ekol. Pol., 19 : 485-497.

Laville (H.). 1971. - Recherches sur les Chironomides (Diptera) lacustres du massif de Néouvielle (Hautes-Pyrénées). $1^{\text {re }}$ partie : systématique, écologie et phénologie. Annls limnol., 7 : 173-332.

Laville (H.). 1971. - Recherches sur les Chironomides (Diptera) lacustres du massif de Néouvielle (Hautes-Pyrénées). $2^{\mathrm{e}}$ partie : communautés et production. Annls limnol., $7: 335-413$.

LENZ (F.). 1931. - Untersuchungen über die Vertikalverteilung der der Bodenfauna in Tiefensedimenten von Seen. Vehr. int. verein. limnol., $5: 232-260$.

Mackereth (F.J.H.). 1963. - Chemical methods for limnologists. Sc. Pub. Freshw. Biol. Ass. n ${ }^{\circ} 21,70$ p.

Mackereth (F.J.H.). 1966. - Some chemical observations on post glacial lake sediments. Phil. Trans. $R$. Soc. (B), $250: 165-213$.

Milbrink (G.). 1969. - Microgradients at the mud-water interface. Inst. Fresh. Res. Drott., $49: 129-148$.

Pugh (T.M.). 1964. - Studies on the vertical distribution of the fauna in the bottom of shallow waters with special reference to insects. Entomologist., 97 : 177-192.

Rey (J.). et Dupin (B.). 1973 a. - Ecologie des Crustacés benthiques du lac de Port-Bielh (Pyrénées centrales). I. Répartition. Annls limnol., 9 : 121-134.

Rex (J.) et Dupin (B.). 1973 b. - Ecologie des Crustacés benthiques du lac de Port-Bielh (Pyrénées centrales). II. Cycles biologiques. Annls limnol., 9 : 259-271.

RyBaK (J. I.). 1969. - Bottom sediments of the lakes of various trophic type. Ekol. Pol. (A), $17: 611-662$.

SärkKä (J.) et PaAsivirta (L.). 1972. - Vertical distribution and abundance of the macro and meiofauna in the profundal sediments of Lake Päijänne, Finland. Ann. Zool. Fenn., 9 : 1-9.

Stockner (J.) et Lund (J.W.G.). 1970. - Live algae in postglacial lake deposits. Limnol. oceanogr., 15: 41-58. 